\title{
Numerical strategy for unbiased homogenization of random materials
}

\author{
R. Cottereau* \\ Laboratoire MSSMat UMR 8579, École Centrale Paris, CNRS, grande voie des vignes, F-92295 Châtenay-Malabry, \\ France
}

\begin{abstract}
SUMMARY
This paper presents a numerical strategy that allows to lower the costs associated to the prediction of the value of homogenized tensors in elliptic problems. This is done by solving a coupled problem, in which the complex microstructure is confined to a small region and surrounded by a tentative homogenized medium. The characteristics of this homogenized medium are updated using a self-consistent approach and are shown to converge to the actual solution. The main feature of the coupling strategy is that it really couples the random microstructure with the deterministic homogenized model, and not one (deterministic) realization of the random medium with a homogenized model. The advantages of doing so are twofold: (a) the influence of the boundary conditions is significantly mitigated, and (b) the ergodicity of the random medium can be used in full through appropriate definition of the coupling operator. Both of these advantages imply that the resulting coupled problem is less expensive to solve, for a given bias, than the computation of homogenized tensor using classical approaches. Examples of 1D and 2D problems with continuous properties, as well as a 2D matrix-inclusion problem, illustrate the effectiveness and potential of the method. Copyright (C) 2013 John Wiley \& Sons, Ltd.
\end{abstract}

Received ...

KEY WORDS: Homogenization; Random material; Arlequin method; self-consistent model; Numerical mesoscope; Representative volume element

\section{INTRODUCTION}

There exist to date fewer theoretical results on the homogenization of random media than of periodic media. Nevertheless, some results, in the case of linear elliptic partial differential equations for example, have shown that one can find a uniform deterministic tensor that produces an accurate approximation of the original solution obtained with the fluctuating stochastic tensor. Such convergence results have been made possible by using the energy method [1] of Tartar [2], by considering the direct construction of the so-called correctors [3], by resorting to strong Gconvergence of operators in a general stochastic setting [4], or by using the $\Gamma$-convergence [5]. Convergence was obtained either in a mean-square sense (for example, in [6, 7] or [1]) or in an almost-sure sense (for example in [3]). Later on, more complex equations were also treated, and weaker hypotheses on the random fields introduced (see for instance $[8,9,10,11,12]$ ).

However, the actual computation of the value of this effective tensor is not always a simple task, besides some particular cases for which analytical (1D problems in particular) or specific numerical solutions are available (see for instance $[13,14]$ in a random quasi-periodic setting).

*Correspondence to: regis.cottereau@ecp.fr

Contract/grant sponsor: ANR project TYCHE (Advanced methods using stochastic modeling in high dimension for uncertainty modeling, quantification and propagation in computational mechanics of solids and fluids) and DIGITEO Région Ile-de-France; contract/grant number: ANR-2010-BLAN-0904 and 2009-26D 
Indeed, the prediction of the effective tensor involves the solution of a corrector problem which is a priori posed on a domain of infinite size. In order to approximate the effective tensor through numerical simulations, the domain therefore has to be truncated at some finite distance and boundary conditions to be introduced. For these bounded domains, the estimated tensor is then a random variable, the variance of which goes to zero when the size of the domain is increased. It has been proved $[8,15]$ that, whatever the choice of boundary conditions, the limit of the estimated tensors was indeed the effective tensor. However, convergence with respect to the size of the domain may be very slow. Alternatively, it is also possible $($ see $[8,16])$ to use a smaller domain and perform averages over several realizations of the random medium. Several authors have followed this path (see for instance $[17,18,19,20]$ ), even putting up schemes to accelerate convergence (through angular averaging in [21] among others, or through the use of antithetic variables in [22]). However, it has been observed that, even though these schemes converge, they do so to biased values. Further, these biases only cancel when the size of the domain becomes very large (with respect to the correlation length).

This paper presents a numerical strategy to identify the homogenized tensor of a random medium. It allows to extend the size of the domain in a cost-effective manner and to play simultaneously with the size of the domain and the discretization along the random dimension (number of Monte Carlo samples) to yield the effective tensor. This is achieved through the coupling of the random microstructure with a homogenized macrostructure, the characteristics of which are updated iteratively using a self-consistent approach. Using this coupled approach, the size of the complex microstructure is limited, while the boundary conditions are pushed away and their influence limited through the tentative homogenized medium. The main feature of the coupling strategy is that it really couples the random microstructure with the deterministic homogenized model, and not each (deterministic) realization of the random medium with a homogenized model, in a fully independent manner. Hence, the ergodicity of the random medium can be used in full to accelerate convergence and minimize the bias introduced by the finite size of the domain.

The idea of coupling the microstructure to a homogenized medium to limit the influence of the boundary conditions was already developed in [23] and [24], but with three major differences: (1) the microstructure is here random, while it was deterministic (and heterogeneous) in the previous papers, (2) the coupling is here made over a volume rather than along a surface, and (3) the approach is coupled to an iterative scheme in order to identify the value of the effective tensor, while it was previously only used to perform direct computations, for a given value of the homogenized tensor.

In Section 2 of the paper, the random medium and model equation that we consider are described in detail, and the classical Dirichlet and Neumann homogenization schemes are presented. In Section 3, we briefly recall the main ingredient of our approach, which is the deterministic-stochastic coupling scheme, previously described in $[25,26]$. Section 4 concentrates on the main novelty of this paper, which is the iterative technique to derive the homogenized tensor. Finally, the last section presents a series of $1 \mathrm{D}$ and $2 \mathrm{D}$ experiments to demonstrate the effectiveness and potential of the proposed approach. Concluding remarks are provided in Section 6.

Throughout the paper, we will use bold characters for random quantities, lowercase characters for scalars and vectors, and uppercase characters for matrices and tensors.

\section{HOMOGENIZATION OF A RANDOM MICROSTRUCTURE}

In this section, we describe the random medium for which we intend to find the homogenized effective properties. We also recall some definitions related to the homogenization of the heat equation.

\subsection{Definition of the model and hypotheses on the random field}

Let us introduce a domain $D \in \mathbb{R}^{d}$, with a typical length scale $L$, a (deterministic) loading field $f(x)$ and a field $\boldsymbol{u}(x)$ governed by the heat equation: find $\boldsymbol{u}(x) \in \mathcal{L}^{2}\left(\Theta, \mathcal{H}^{1}(D)\right)$ such that, $\forall x \in D$, almost surely:

$$
-\nabla \cdot(\boldsymbol{k}(x) \nabla \boldsymbol{u}(x))=f(x),
$$


for a random field $\boldsymbol{k}(x)$ fluctuating over a length scale $\ell_{c}$ (usually defined through the correlation length), and with appropriate boundary conditions. Here, $(\Theta, \mathcal{F}, P)$ is a complete probability space, with $\Theta$ a set of outcomes, $\mathcal{F}$ a $\sigma$-algebra of events in $\Theta$, and $P: \mathcal{F} \rightarrow[0,1]$ a probability measure.

In order to obtain a homogenized material, the random parameter field $\boldsymbol{k}(x)$ is required to verify certain hypotheses. In particular, it is assumed to be bounded and uniformly coercive, that is to say $\exists \kappa_{m}, \kappa_{M} \in(0,+\infty)$, such that

$$
0<\kappa_{m} \leq \boldsymbol{k}(x) \leq \kappa_{M}<\infty, \forall x \in D, \text { almost surely. }
$$

Also, it is required to be stationary and ergodic.

\subsection{Definition of homogenization}

Homogenization deals with cases when the ratio $\epsilon=\ell_{c} / L$ is small. We then scale the fluctuations of the microstructure by $1 / \epsilon$, and look at the fluctuations of the solution $\boldsymbol{u}(x)$ at the original scale. The following sequence of problems is therefore considered: find $\boldsymbol{u}_{\epsilon}(x) \in \mathcal{L}^{2}\left(\Theta, \mathcal{L}^{2}(D)\right)$ such that, $\forall x \in D$, almost surely:

$$
-\nabla \cdot\left(\boldsymbol{k}_{\epsilon}(x) \nabla \boldsymbol{u}_{\epsilon}(x)\right)=f(x),
$$

where $\boldsymbol{k}_{\epsilon}(x)=\boldsymbol{k}(x / \epsilon)$, and with appropriate boundary conditions, for instance $\boldsymbol{u}_{\epsilon}(x)=0, \forall x \in \partial D$ (see Subsection 2.3 for the definition of Dirichlet and Neumann approximations of the homogenized coefficients). Under suitable hypotheses, in particular on the random field $\boldsymbol{k}_{\epsilon}(x)$ (described in the previous Subsection 2.1), each of these problems admits a unique solution.
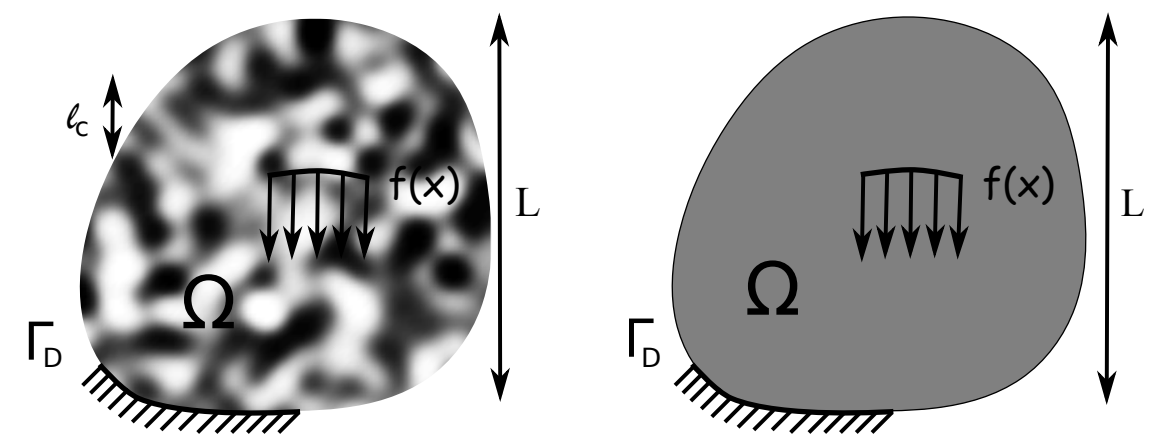

Figure 1. Description of one realization of the random medium (left), with fluctuating coefficient $\boldsymbol{k}_{\epsilon}(x)$, and corresponding effective medium (right), with constant deterministic effective tensor $K^{*}$.

Using different sets of hypotheses and with different methods, many authors (see the references provided in the introduction) have shown that, independently of the load $f(x)$, the sequence of solutions $\boldsymbol{u}_{\epsilon}(x)$ converges when $\epsilon \rightarrow 0$ to the solution $u^{*}(x)$ of the following deterministic problem: find $u^{*}(x)$ such that, $\forall x \in D$ :

$$
-\nabla \cdot\left(K^{*} \nabla u^{*}(x)\right)=f(x),
$$

with corresponding boundary conditions. A priori, the effective coefficient $K^{*}$ is a full second-order tensor, meaning that the homogenized material potentially exhibits anisotropy.

The constructive definition of the effective tensor requires the solution of the so-called corrector problem, which states: find $\boldsymbol{w}_{\epsilon}(x)$ such that, $\forall x \in D$, almost surely:

$$
-\nabla \cdot\left(\boldsymbol{k}_{\epsilon}(x)\left(I+\nabla \boldsymbol{w}_{\epsilon}(x)\right)\right)=0 .
$$

As $\boldsymbol{w}_{\epsilon}$ is a vector, $\nabla \boldsymbol{w}_{\epsilon}(x)$ is a tensor, and this equation is a $d$-dimensional equation. The tensor $I$ is the identity tensor in $\mathbb{R}^{d} \times \mathbb{R}^{d}$. The homogenized tensor is then defined as:

$$
K^{*}=\lim _{\epsilon \rightarrow 0} \mathrm{E}\left[\left(I+\nabla \boldsymbol{w}_{\epsilon}(x)\right)^{T} \boldsymbol{k}_{\epsilon}(x)\left(I+\nabla \boldsymbol{w}_{\epsilon}(x)\right)\right] .
$$

Note that, in the limit when $\epsilon \rightarrow 0$, the tensor $K^{*}$ does not depend on the position. 


\subsection{Numerical estimation of homogenized tensor}

For $\epsilon \rightarrow 0$, the corrector equation (5) is either set in a domain of infinite size or with infinitely small details. Also, the mathematical expectation $\mathrm{E}[\cdot]$ in equation (6) is an integral operator over an infinite-dimensional space. Approximations of $K^{*}$ are then constructed by performing at the same time a truncation of $D$ (hence bounding $\epsilon$ to a finite value), introducing particular boundary conditions at the boundary $\partial D$ of the domain, and replacing the mathematical expectation in the evaluation of the homogenized tensor by a sum over a finite number of Monte Carlo samples.

The Kinematic Uniform Boundary Conditions (KUBC) approach consists in using homogeneous Dirichlet boundary conditions at the boundary $\left(\boldsymbol{w}_{\epsilon}=\mathbf{0}, \forall x \in \partial D\right.$, almost surely), and approximating the homogenized tensor, hereafter denoted $\check{K}_{N}^{\epsilon}$, by

$$
\check{K}_{N}^{\epsilon}=\frac{1}{N|D|} \sum_{i=1}^{N} \int_{D}\left(I+\nabla w_{\epsilon}^{i}(x)\right)^{T} k_{\epsilon}^{i}(x)\left(I+\nabla w_{\epsilon}^{i}(x)\right) d x,
$$

where $|D|=\int_{D} d x$, the $k_{\epsilon}^{i}(x)$ are realizations of the stochastic field $\boldsymbol{k}_{\epsilon}(x)$ and the $w_{\epsilon}^{i}(x)$ are the solution of the corresponding (deterministic) corrector problems, posed over the truncated domain $D$ with finite $\epsilon$, and with the chosen set of boundary conditions. More details on the derivation of this formula can be found in [16, Eq. (16)] or [27, Eq. (5.8)].

The Static Uniform Boundary Conditions (SUBC) approach consists in using Neumann boundary conditions $\left(\boldsymbol{k}_{\epsilon}(x)\left(I+\nabla \boldsymbol{w}_{\epsilon}\right) \cdot n=I \cdot n, \forall x \in \partial D\right.$, almost surely), and approximating the homogenized tensor, hereafter denoted $\hat{K}_{N}^{\epsilon}$, by

$$
\hat{K}_{N}^{\epsilon}=\left[\frac{1}{N|D|} \sum_{i=1}^{N} \int_{D}\left(I+\nabla w_{\epsilon}^{i}(x)\right)^{T} k_{\epsilon}^{i}(x)\left(I+\nabla w_{\epsilon}^{i}(x)\right) d x\right]^{-1} .
$$

More details on the derivation of this formula can be found in [16, Eq. (16)] or [27, Eq. (5.9)].

A very efficient alternative to these two techniques consists in using periodic boundary conditions (see for instance [28] for mathematical details, or [29] for an efficient FFT implementation of this technique). This method works very well, but it requires, on the other hand, that the microstructure be itself periodic. Its application in the context of random media therefore requires some hypotheses on the correlation structure, or a modification of the distribution for periodization. Comparison of periodic, SUBC and KUBC estimates to the method that we propose in this paper will be made in the applications (see in particular Section 5.3).

Note that the tensors $\check{K}_{N}^{\epsilon}$ and $\hat{K}_{N}^{\epsilon}$ (as well as any other obtained through a similar approach with other boundary conditions) depend obviously on both the number $N$ of Monte Carlo samples that are used to approximate the mathematical expectation and on the value of $\epsilon$. They also depend on the boundary conditions that were used to approximate the corrector problems and are therefore $a$ priori different one from the other. For elliptic equations, the influence of these boundary conditions disappears for $\epsilon \rightarrow 0$ (see the proof for the KUBC, SUBC, and periodic boundary conditions in [15]), but may become extremely important for small domains (see the examples in Section 5).

\subsection{Particular case in $1 D$}

The 1D case is very particular, in the sense that the corrector problem can be solved analytically, whatever the choice of probability law for $\boldsymbol{k}_{\epsilon}$. The homogenized tensor (in that case a scalar) is then:

$$
K^{*}=\mathrm{E}\left[\boldsymbol{k}_{\epsilon}^{-1}\right]^{-1}=\lim _{\epsilon \rightarrow 0}\left(\int_{D}\left(\boldsymbol{k}_{\epsilon}(x)\right)^{-1} d x\right)^{-1} .
$$

It is interesting to note that the KUBC and SUBC approximates can also be computed for any ratio $\epsilon$. Indeed, simple algebraic manipulation yields

$$
\check{K}_{\infty}^{\epsilon}=\mathrm{E}\left[\boldsymbol{K}_{D}\right]
$$


where $\boldsymbol{K}_{D}=|D| / \int_{D} \boldsymbol{k}_{\epsilon}^{-1}(x) d x$ and

$$
\hat{K}_{\infty}^{\epsilon}=\mathrm{E}\left[\boldsymbol{K}_{D}^{-1}\right]^{-1}=K^{*} .
$$

When $\epsilon \rightarrow \infty$ the random field $\boldsymbol{k}_{\epsilon}(x)$ becomes, at the scale of the domain $D$, a random variable (with no fluctuation in space). Hence, we have $\boldsymbol{K}_{D}=\boldsymbol{k}_{\epsilon}$, and $\check{K}_{\infty}^{\infty}=\mathrm{E}\left[\boldsymbol{k}_{\epsilon}\right]$, and this quantity does not depend on the position $x$ thanks to the hypothesis of stationarity of the field. When $\epsilon \rightarrow 0$, the ergodicity hypothesis on the random field and the definition of $\boldsymbol{K}_{D}$ yield the expected result of equation (9) $\check{K}_{\infty}^{0}=K^{*}$. In general, when $\epsilon \neq 0$, we have $\check{K}_{\infty}^{\epsilon} \neq K^{*}$. This means that the KUBC estimate is different from the homogenized coefficient, even though an infinite number of Monte Carlo trials is considered. In this paper, we will refer to this misfit by the word "bias".

It is interesting to note that in 1D, whatever the value of $\epsilon$, the SUBC approach yields the exact value of the homogenized tensor (when $N \rightarrow \infty$ ). In the words defined above, the SUBC estimate in $1 \mathrm{D}$ is therefore unbiased. Note, however, that this property is very specific to the one-dimensional case, and is not true in higher dimensions.

\subsection{Particular case in $2 D$}

We discuss in this section a very particular type of $2 \mathrm{D}$ medium that has a specific kind a duality property: the random field $\boldsymbol{k}_{\epsilon}(x)$ is statistically equivalent to the random field $c / \boldsymbol{k}_{\epsilon}(x)$, where $c$ is a scalar constant. As noted in [30, chapter 3] (see a proof in the book in a more general setting, and references therein for original contributions to that result), the homogenized coefficient of this random medium is then necessarily equal to

$$
K^{*}=\sqrt{c} I_{2}
$$

where $I_{2}$ is the two-dimensional identity tensor.

Note that, in the two-dimensional case, there is no analytic result for the value of the KUBC and SUBC homogenized approximates at finite $\epsilon$. However, the following bounds always hold true (see [27] for example):

$$
\hat{K}_{\infty}^{\epsilon} \leq K^{*} \leq \check{K}_{\infty}^{\epsilon} .
$$

Further, as we will be illustrated in the examples at the end of this paper, both the KUBC and SUBC are biased for finite $\epsilon$ (see Section 5).

\section{COUPLING OF A RANDOM MICROSTRUCTURE WITH AN EFFECTIVE MODEL}

In the previous section, we have introduced classical numerical techniques to obtain estimates of the homogenized tensors. These estimates are widely developed and used in the literature, but are unfortunately biased in the general case. In this paper, we propose a novel technique for obtaining such estimates. This technique will be presented in Section 4 and relies heavily on a stochasticdeterministic coupling approach originally introduced in [25, 26]. The objective of this section is to recall the main features of this coupling method, without too much emphasis on technical details (those can be found in particular in [26]).

It is important to stress from the start that this method is very different from an approach where a sequence of realizations of the random medium would be coupled each to an exterior effective model. In such an approach, the displacement fields in the effective model would be different for each realization of the random medium. Contrarily, in our approach, the coupling is really posed in a stochastic setting and couples the entire set of realizations of the random medium to a single effective model.

This coupling strategy is based on the introduction and superposition of two models and three domains (see figure 2): the (stochastic) microstructure is defined over a domain $D$ with a stochastic parameter field $\boldsymbol{k}_{\epsilon}(x)$, and the (deterministic) effective model is defined over a domain $\underline{D}$, with a constant parameter $\underline{K}_{\epsilon}$. The supports of the two models are such that $D \subset \underline{D}$, and the two models communicate through a coupling volume $D_{c}$, with $D_{c} \subset D$ and $D_{c} \subset \underline{D}$. These definitions mean 
that there is part of the domain where only the effective model is defined, part of the domain where both models are defined and over which they are coupled, and part of the domain where both models are defined but over which they do not communicate.
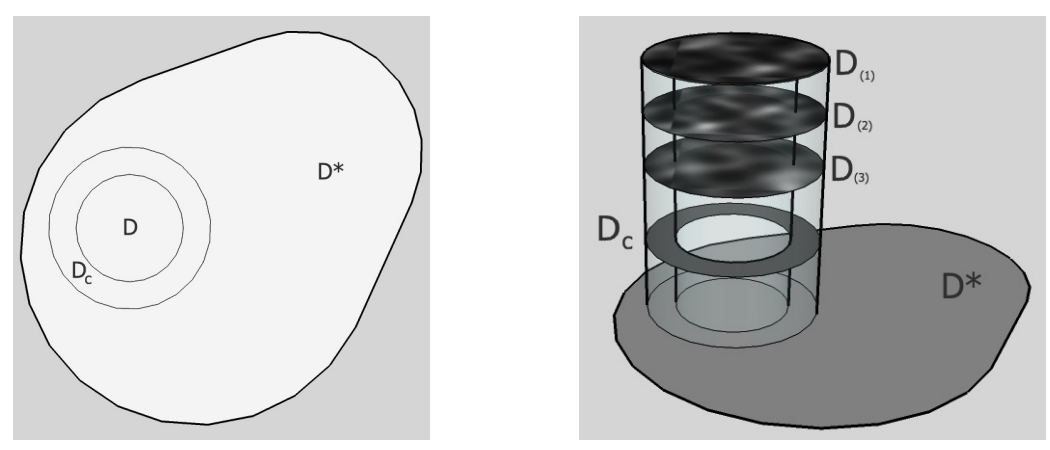

Figure 2. Section (left) and perspective view (right) of a 2D Arlequin problem where an effective model, defined over domain $\underline{D}$, and several realizations of a random model, defined over $D$, are coupled through a coupling domain $D_{c}$. On the overlap outside of $D_{c}:(D \cap \underline{D}) \backslash D_{c}$, both models are defined but behave independently.

The coupling problem is set in the general Arlequin framework (see in particular [31, 32, 33, 34] for details on the Arlequin framework in a deterministic setting and $[25,26]$ for the stochastic case), and reads: find $\left(\underline{u}_{\epsilon}, \boldsymbol{u}_{\epsilon}, \boldsymbol{\Phi}\right) \in \underline{\mathcal{V}} \times \mathcal{W} \times \mathcal{W}_{c}$ such that

$$
\left\{\begin{array}{ll}
a_{\epsilon}\left(\underline{u}_{\epsilon}, v\right)+C(\boldsymbol{\Phi}, v)=\ell(v), & \forall v \in \mathcal{V} \\
\mathcal{A}_{\epsilon}\left(\boldsymbol{u}_{\epsilon}, \boldsymbol{v}\right)-C(\boldsymbol{\Phi}, \boldsymbol{v})=\mathcal{L}(\boldsymbol{v}), & \forall \boldsymbol{v} \in \mathcal{W} \\
C\left(\boldsymbol{\Psi}, \underline{u}_{\epsilon}-\boldsymbol{u}_{\epsilon}\right)=0, & \forall \boldsymbol{\Psi} \in \mathcal{W}_{c}
\end{array},\right.
$$

where the forms $a_{\epsilon}$ and $\ell$, on the one hand, and $\mathcal{A}_{\epsilon}$ and $\mathcal{L}$, on the other hand, are the forms appearing in the weak formulations corresponding to equations (4) and (1), respectively, weighted by a function that enforces the conservation of the global energy, by appropriate partitioning among the two available models. More specifically, these forms are:

$$
\begin{gathered}
a_{\epsilon}(u, v)=\int_{\underline{D}} \alpha_{1}(x) \underline{K}_{\epsilon} \nabla u \cdot \nabla v d x, \\
\ell(v)=\int_{\underline{D}} \alpha_{1}(x) f(x) v d x, \\
\mathcal{A}_{\epsilon}(\boldsymbol{u}, \boldsymbol{v})=\mathrm{E}\left[\int_{D} \alpha_{2}(x) \boldsymbol{k}_{\epsilon}(x) \nabla \boldsymbol{u} \cdot \nabla \boldsymbol{v} d x\right],
\end{gathered}
$$

and

$$
\mathcal{L}(\boldsymbol{v})=\int_{D} \alpha_{2}(x) f(x) \mathrm{E}[\boldsymbol{v}] d x .
$$

These weight functions mainly mean to split appropriately the total energy among the two models. Therefore, they verify the following constraints: $\alpha_{1}(x)+\alpha_{2}(x)=1$ in $D \cap \underline{D}$ and $\alpha_{1}(x)=1$ in $\underline{D} \backslash D$. Further, they allow to put emphasis on one or the other of the two models. Hence, where the stochastic (fine scale) model is defined (and outside of the coupling area), the weight function $\alpha_{2}(x)$ is given a value close to one (and $\alpha_{1}(x)$ a value close to zero).

The coupling operator $C$ enforces weakly the equality of the two fields $\boldsymbol{u}$ and $\underline{u}$ in the coupling area $D_{c}$. It is defined by:

$$
C(\boldsymbol{u}, \boldsymbol{v})=\mathrm{E}\left[\int_{D_{c}}\left(\kappa_{0} \boldsymbol{u} \boldsymbol{v}+\kappa_{1} \nabla \boldsymbol{u} \cdot \nabla \boldsymbol{v}\right) d x\right] .
$$


In this definition, the constant parameters $\kappa_{0}$ and $\kappa_{1}$ are introduced to weight relatively the $L^{2}$ and $H^{1}$ parts of the scalar product, and the solution of the Arlequin coupled problem is very robust with respect to their choice (see [34]). Note that, in the last line of Equation (14), the second input of the coupling operator is $\underline{u}_{\epsilon}-\boldsymbol{u}_{\epsilon}$, which is formally the subtraction between elements of two different functional spaces $\mathcal{V}$ and $\mathcal{W}$. We actually consider here that $\mathcal{H}^{1}\left(D_{c}\right)$ is naturally embedded in $\mathcal{L}^{2}\left(\Theta, \mathcal{H}^{1}\left(D_{c}\right)\right.$, so that, over $D_{c}$ this subtraction makes sense. For more complex cases where the models are not related in such a simple manner, projection operators have to be introduced (see for example [35]).

The functional spaces are $\underline{\mathcal{V}}=\mathcal{H}_{0}^{1}(\underline{D})$ (with straightforward modifications for other boundary conditions than homogeneous Dirichlet around $\underline{D}), \mathcal{W}=\mathcal{L}^{2}\left(\Theta, \mathcal{H}^{1}(D)\right)$ (assuming that $D$ is strictly embedded in $\underline{D}$ ), and

$$
\begin{aligned}
\mathcal{W}_{c} & =\mathcal{H}^{1}\left(D_{c}\right) \oplus \mathcal{L}^{2}(\Theta, \mathbb{R}) \\
& =\left\{\psi(x)+\boldsymbol{\theta} \mathbb{I}_{c}(x) \mid \psi \in \mathcal{H}^{1}\left(D_{c}\right), \boldsymbol{\theta} \in \mathcal{L}^{2}(\Theta, \mathbb{R})\right\}
\end{aligned}
$$

The indicator function $\mathbb{I}(x)$ is such that $\mathbb{I}_{c}\left(x \in D_{c}\right)=1$ and $\mathbb{I}_{c}\left(x \notin D_{c}\right)=0$. Hence the mediator space $\mathcal{W}_{c}$ can be seen as composed of functions with a spatially-varying ensemble average and perfectly spatially-correlated randomness. The random part is actually the generator of the kernel of the acoustic operator we are considering here. If we were to consider a vector equation, the definition of the mediator space would be the superposition of a space-fluctuating average (as here), and six rigid-body movements with random coefficients.

Note that, thanks to the specific structure of the space $\mathcal{W}_{c}$, the last equality of the system (14) can be written equivalently, $\forall \boldsymbol{\Psi}=\psi(x)+\boldsymbol{\theta} \mathbb{I}_{c}(x) \in \mathcal{W}_{c}$, or otherwise said, $\forall \psi \in \mathcal{H}^{1}\left(D_{c}\right)$ and $\forall \boldsymbol{\theta} \in \mathcal{L}^{2}(\Theta, \mathbb{R})$,

$$
\begin{aligned}
0 & =C\left(\boldsymbol{\Psi}, \underline{u}_{\epsilon}-\boldsymbol{u}_{\epsilon}\right) \\
& =\mathrm{E}\left[\int_{\Omega_{c}}\left(\kappa_{0}\left(\psi+\boldsymbol{\theta} \mathbb{I}_{c}\right)\left(\underline{u}_{\epsilon}-\boldsymbol{u}_{\epsilon}\right)+\kappa_{1} \nabla \psi \cdot \nabla\left(\underline{u}_{\epsilon}-\boldsymbol{u}_{\epsilon}\right)\right) d x\right] \\
& =C\left(\mathrm{E}[\boldsymbol{\Psi}], \underline{u}_{\epsilon}-\mathrm{E}\left[\boldsymbol{u}_{\epsilon}\right]\right)-\mathrm{E}\left[\boldsymbol{\theta} \int_{\Omega_{c}}\left(\boldsymbol{u}_{\epsilon}-\mathrm{E}\left[\boldsymbol{u}_{\epsilon}\right]\right) d x\right] .
\end{aligned}
$$

Therefore, this condition imposes that, in each space point $x \in D_{c}$, the (ensemble) average of the random field $\mathrm{E}\left[\boldsymbol{u}_{\epsilon}(x)\right]$ should be equal to the field $\underline{u}_{\epsilon}(x)$, and that the variability of the spaceaveraged random variable $\int_{\Omega_{c}}\left(\boldsymbol{u}_{\epsilon}-\mathrm{E}\left[\boldsymbol{u}_{\epsilon}\right]\right) d x$ should cancel.

The stability of the coupled problem (14) was proved in [26], and its solution can be provided either by Monte Carlo sampling of the random space, or by a spectral approach.

\section{A NEW METHOD FOR THE DETERMINATION OF THE HOMOGENIZED TENSOR}

In the previous two sections, we have described classical approaches to the numerical homogenization of random structures (Section 2) and a new coupling method between stochastic and deterministic models (Section 3). Although these two sections may have seemed very weakly related, we will show here how the latter method can be used for the design of a novel numerical homogenization technique for random media.

\subsection{Principle of the method}

The general motivation for the design of this technique lies in the observation that the biases observed in the SUBC and KUBC estimates of the homogenized coefficients originate from the boundary conditions chosen for each realization of the random corrector problems. Somehow, in order to obtain good estimates of the homogenized coefficients, these boundary conditions have to be taken away (by reducing $\epsilon$ ), in order to minimize their influence.

Some authors (see $[23,24]$ ) have intended to do so by intercalating between the heterogeneous model of interest and the boundary conditions a homogeneous medium, with the appropriate 
homogenized tensor. As the properties of the medium do not vary in space, the corresponding mesh does not need to be heavily refined, and the boundary conditions can therefore be pushed away in a cost-effective manner. If the tentative medium is indeed the homogenized medium corresponding to the heterogeneous fine scale model, then it is expected that the influence of the boundary conditions will be reduced. However, this is not so, because an impedance mismatch has been created at the interface between the two models. Indeed, on this interface, the two models do not have the same mechanical parameters, and this mismatch acts somehow as a new set of virtual boundary conditions. In particular, on one side of the interface, the properties fluctuate, while they are constant on the other side.

The approach that we propose here builds on this initial idea. However, it brings down the above issue by considering a volume coupling, which is much smoother than an interface-based one. Further, it allows to perform the coupling between the entire set of realizations of the random medium and the tentative homogenized medium through the coupling operator described in equation (19).

Apart from this idea of computing a coupled problem, we also introduce an optimization scheme to gather the value of the homogenized tensor, because it is indeed the objective of our work. This optimization scheme builds on the idea that, once the homogenized model has been identified, it should behave exactly the same, whether it is solved alone or coupled to the micro-structure it represents. In particular, the solution of a coupled Arlequin problem with that homogenized model and the micro-structure under the homogenization experiments presented in Section 2.3 should yield exactly the same result as if the homogenized model was solved alone. On the other hand, if the tentative homogenized is not correct, there will be a mismatch of impedances that can be detected through the distance between the response of the homogenized model in the coupled model and that of the same homogenized model solved alone. Hence for an imposed unit strain at the boundary of the macro-scale domain (Dirichlet approach, which we will consider in the following), the strain tensor should be identity, whether the macro-model alone is solved for, or the coupled micro-macro model.

\subsection{Description of the algorithm}

Algorithm 1: Algorithmic description of the proposed iterative technique for numerical homogenization of random materials

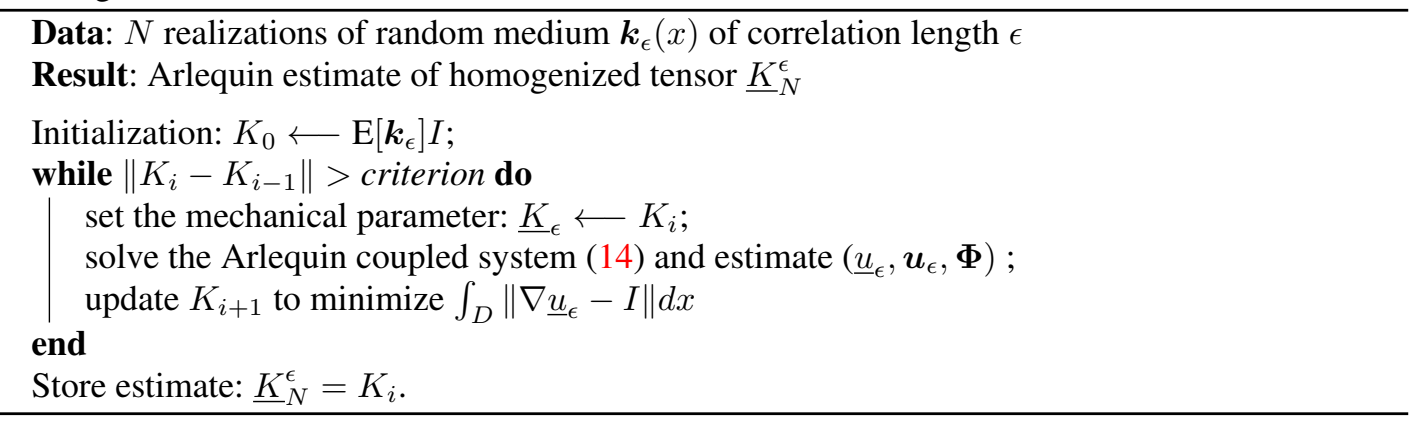

In this algorithm, note that the iterative loop can be efficiently implemented through classical general-purpose optimization schemes. In particular, we have used the Nelder-Mead algorithm (see [36] for details), but others could be considered. Similarly, we chose as initial value $K_{0}=$ $\mathrm{E}\left[\boldsymbol{k}_{\epsilon}\right] I$, but other choices are equally reasonable. The influence of the choice of initial value will be discussed through examples in Section 5.

We have chosen here to drive the iterative scheme with the minimization of the potential $\int_{D}\left\|\nabla \underline{u}_{\epsilon}-I\right\| d x$, consistent with the intuitive idea developed above (Section 4.1). Other possibilities exist. In particular, we have tested the examples in Section 5 with both this potential and the energy norm $\int_{D}\left(\nabla \underline{u}_{\epsilon}-I\right) \cdot \underline{K}_{\epsilon}\left(\nabla \underline{u}_{\epsilon}-I\right) d x$. The results obtained were exactly the same. Here, we prefer to avoid using $\underline{K}_{\epsilon}$ in the definition of the potential, because it is the quantity we are iterating on. However, for other problems, in particular when the homogenized tensor is not scalar anymore, it might become interesting to consider the second type of operator. 


\subsection{Evaluation of numerical costs}

Let us finally discuss the comparative numerical costs between the standard numerical homogenization schemes described in Section 2.3 and the proposal made here. From the point of view of our method, the differences in cost can be listed as:

- degrees of freedom are introduced for the discretization of $\mathcal{V}^{*}$ in (14);

- degrees of freedom are introduced for the discretization of $\mathcal{W}_{c}$ in (14);

- the iterative loop means that several Arlequin systems (14) are solved;

- the estimate of the homogenized tensor is less polluted by boundary conditions.

Basically, our approach becomes interesting when the gain from the last item overcomes the costs induced by the first three. In that respect, it should be noted that the discretization of the functional space $\underline{\mathcal{V}} \subset \mathcal{H}^{1}(\underline{D})$ is very coarse compared to the discretization of $\mathcal{H}^{1}(D)$ because the mechanical properties are constant in the homogenized model while they are heterogeneous over the stochastic model. Likewise, the discretization in space of $\mathcal{W}_{c}=\mathcal{H}^{1}\left(D_{c}\right) \oplus \mathcal{L}^{2}(\Theta, \mathbb{R})$ can be made to follow that of $\mathcal{H}^{1}(\underline{D})$, and therefore introduce very few additional degrees of freedom. Finally, concerning the iterative scheme, it should be noted that as the realizations of the random model do not change between two iterations (only the homogenized model evolves), the assembly of the Monte Carlo samples of stiffness matrices does not have to be repeated.

\section{APPLICATIONS}

In this section, we consider the implementation of our homogenization approach on two problems for which analytical solutions are available and one classical problem in periodic homogenization. The software used for the solution of the coupled Arlequin systems is freely available at https://github.com/cottereau/CArl.

In all the simulations presented in this section, we have used $\kappa_{0}=1$ and $\kappa_{1}=10^{-3}$ for the definition of the coupling operator (see Eq. (19)). Also, we have used $\alpha_{2}\left(x \in(\underline{D} \cap D) \backslash D_{c}\right)=$ $1-\eta$ and $\alpha_{1}\left(x \in(\underline{D} \cap D) \backslash D_{c}\right)=\eta$, with $\eta=10^{-3}$, for the weighting of the energies of the two models. The realizations of the continuous random fields $\boldsymbol{k}_{\epsilon}(x)$ have been generated using the spectral representation method [37], and its Fast Fourier Transform implementation. Finally, in the implementation of the loop in algorithm 1 , a relative tolerance of criterion $=10^{-2}$ was selected for both the value and the argument of the potential function.

\subsection{D isotropic medium with random continuous properties}

In this first example, we show the effectiveness of the method and discuss numerical implementation details.

5.1.1. Description of the model. We consider a two-dimensional problem, within a domain $D=$ $[0,1] \times[0,1]$, of typical size $L=1$. The operator to be homogenized is $\nabla \cdot \boldsymbol{k}_{\epsilon}(x) \nabla \boldsymbol{u}_{\epsilon}$, with $\boldsymbol{k}_{\epsilon}(x)$ a random heterogeneous modulus. We consider for $\boldsymbol{k}_{\epsilon}(x)$ a homogeneous random field with lognormal first-order marginal distribution, and average and standard deviation $\mathrm{E}\left[\boldsymbol{k}_{\epsilon}\right]=\sigma=\sqrt{2}$. The power spectrum is considered triangular (which corresponds to a square cardinal sine correlation), with correlation length $\ell_{c}$. We will consider the homogenization problem for three different relative correlation lengths: $\epsilon=\ell_{c} / L=10, \epsilon=1$ and $\epsilon=0.1$. These correlation lengths span several orders of magnitude (see Figure 3 for examples of realizations of the random media considered) in order to show a wide range of behaviors for our method. Note that considering a domain of fixed size and variable correlation lengths, as is done here, is strictly equivalent to considering a constant random field homogenized over cells of variable sizes, as is more often done in the micro-mechanical community.

As discussed in Section 2.5, the homogenized tensor is

$$
K^{*}=\left[\begin{array}{ll}
1 & 0 \\
0 & 1
\end{array}\right]
$$




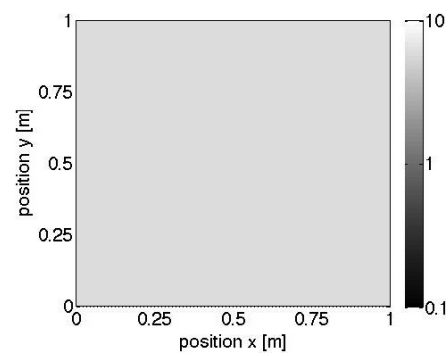

(a) $\epsilon=10$, Sample 1

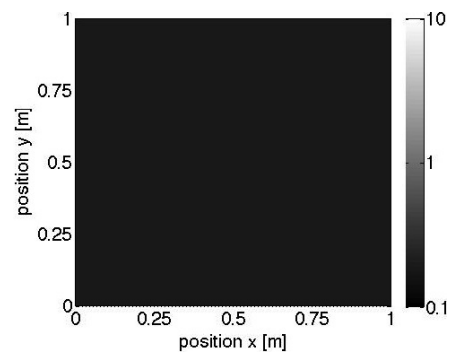

(d) $\epsilon=10$, Sample 2

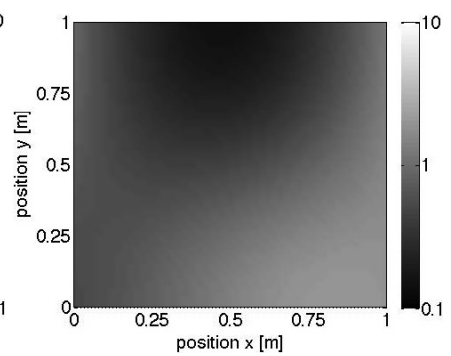

(b) $\epsilon=1$, Sample 1

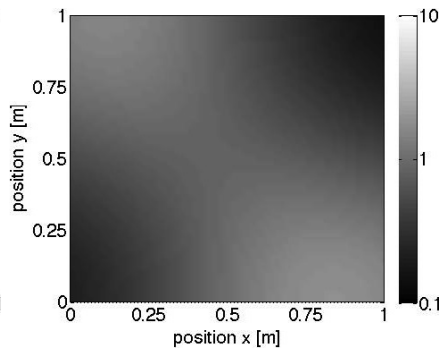

(e) $\epsilon=1$, Sample 2

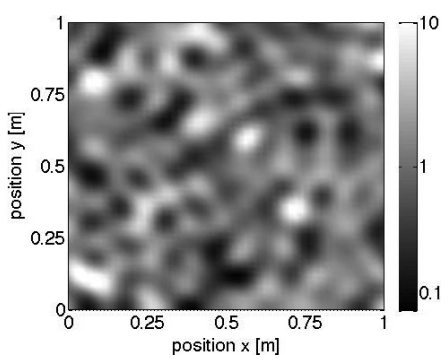

(c) $\epsilon=0.1$, Sample 1

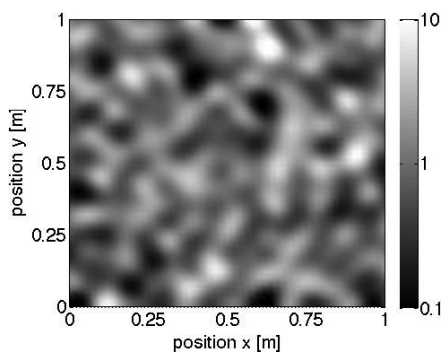

(f) $\epsilon=0.1$, Sample 2

Figure 3. Map of parameter $\boldsymbol{k}_{\epsilon}(x)$ (in logarithmic scale) for two realizations of each of the cases considered here (left: $\epsilon=\ell_{c} / L=10$, center: $\epsilon=1$, and right: $\epsilon=0.1$ ).

Indeed, the inverse of a log-normal random variable follows exactly the same law as the variable itself in the case when the mean and the standard deviation are equal. Note that, because we know beforehand that the homogenized tensor is isotropic, we will only discuss here the convergence toward the scalar value $K_{x x}^{*}=1$. This means that the KUBC, SUBC, and Arlequin estimates will really be based only on numerical experiments in one direction (using only the imposed gradient $\nabla u_{\epsilon}=\left[\begin{array}{ll}1 & 0\end{array}\right]^{T}$ for the KUBC estimate for instance). More complex cases, with anisotropic homogenized behavior in particular, will be considered in the future.

5.1.2. Computation of KUBC and SUBC estimates. First, we consider the KUBC and SUBC estimates of the homogenized coefficient, as described in Section 2.3. To observe the convergence with respect to the number $N$ of realizations of the random medium over which averages are taken (equations (7) and (8)), we compute KUBC and SUBC estimates for different values of this number $N$. Note that, for a given correlation length, the values of the KUBC and SUBC estimates depend not only on the number $N$, but also on the realizations themselves. We therefore compute, for each value of $N, n=10$ different estimates for different ensembles of $N$ realizations of the random medium. These results are plotted in Figure 4. The linear Finite Element method was used to compute the corrector problems, with 800,1600 , and 10000 triangular elements, respectively for the cases $\epsilon=\ell_{c} / L=10, \epsilon=1$ and $\epsilon=0.1$.

On these plots, we retrieve the expected asymptotic behavior of the homogenized coefficients. Both the KUBC $\check{K}_{N}^{\epsilon}$ and SUBC $\hat{K}_{N}^{\epsilon}$ estimates converge to the exact value $K^{*}$ for small $\epsilon$ and large $N$ (although the KUBC is not fully converged at $\epsilon=0.1$ ). Also, at large $\epsilon$ and large $N$, the KUBC estimate tends towards the arithmetic average $\mathrm{E}\left[\boldsymbol{k}_{\epsilon}\right]$ and the SUBC estimate tends towards the harmonic average $\mathrm{E}\left[\boldsymbol{k}_{\epsilon}^{-1}\right]^{-1}$. Finally, for a fixed $\epsilon$ and increasing $N$, the variances of both the KUBC and SUBC estimates decrease, canceling for $N \rightarrow \infty$. Likewise, the variances of these estimates decrease for fixed $N$ and decreasing $\epsilon$, canceling again for $\epsilon \rightarrow 0$.

5.1.3. Computation of the Arlequin estimate. We now turn to the estimation of the Arlequin estimate $\underline{K}_{N}^{\epsilon}$ of the homogenized coefficient over domains $\underline{D}=[-1 ; 2] \times[-1 ; 2]$ and $D=[0 ; 1] \times$ 


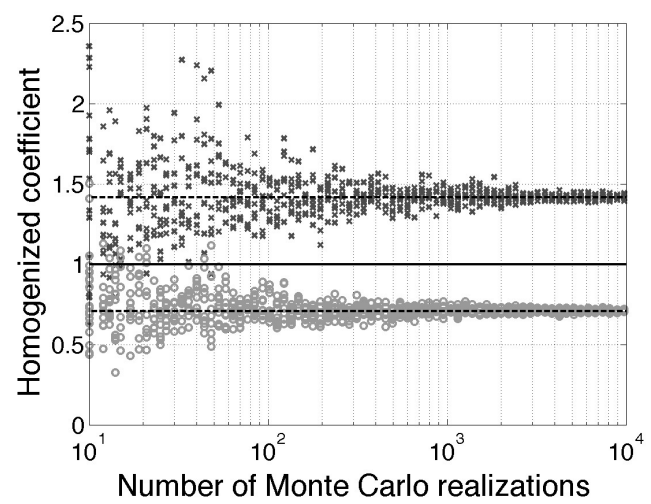

(a) $\epsilon=10$

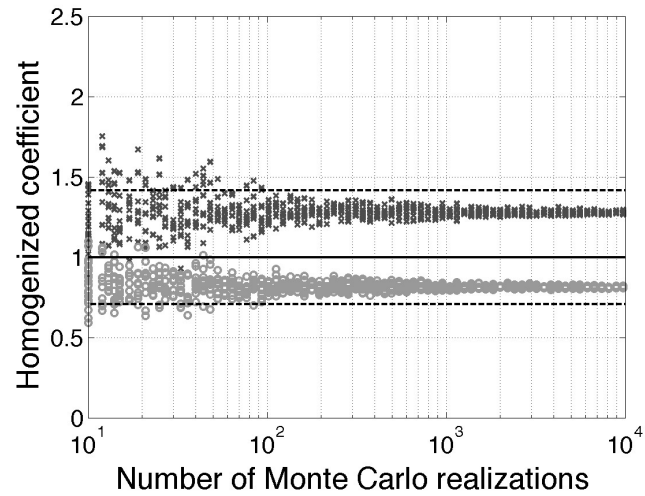

(b) $\epsilon=1$

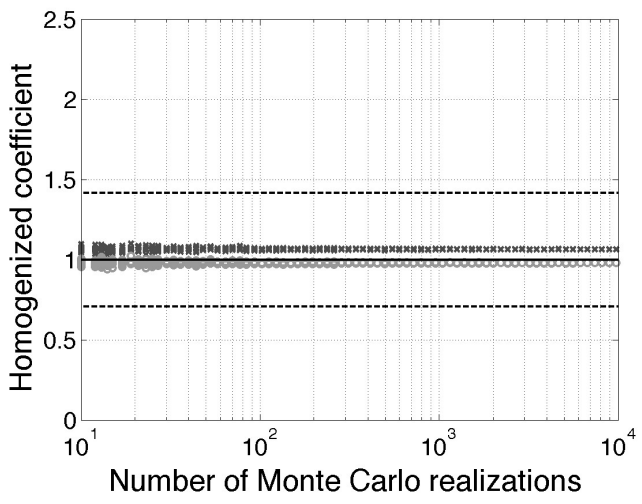

(c) $\epsilon=0.1$

Figure 4. Convergence of the homogenized coefficients $\check{K}_{N}^{\epsilon}$ (dark grey crosses) and $\hat{K}_{N}^{\epsilon}$ (light grey circles) for different correlation lengths ( (a) $\epsilon=\ell_{c} / L=10$, (b) $\epsilon=1$, and (c) $\epsilon=0.1$ ) as a function of the numbers of Monte Carlo trials $N$. The dashed lines indicate the values of the arithmetic average $\mathrm{E}\left[\boldsymbol{k}_{\epsilon}\right]$ and of the harmonic average $\mathrm{E}\left[\boldsymbol{k}_{\epsilon}^{-1}\right]^{-1}$. The solid lines indicate the value of $K^{*}=1$.

$[0 ; 1]$. The coupling zone $D_{c}$ is a band of width 0.2 circling at the boundary of $D$, and we consider a unit strain boundary condition at the boundary of $\underline{D}$.

In the Arlequin coupled problem, there exist the same typical lengths as before $\left(\ell_{c}\right.$ and $\left.L\right)$, plus an additional one, corresponding to the size $\underline{L}=3$ of the tentative homogeneous medium $\underline{D}$. In order to simplify the comparisons between the Arlequin estimate and the KUBC/SUBC estimates, we continue to define $\epsilon$ as the ratio of the correlation length $\ell_{c}$ to the size $L$ of the random cell $D$, that actually indicates the amount of statistical information available about the random medium.

In Figure 5, we plot the values of the Arlequin estimates $\underline{K}_{N}^{\epsilon}$ for three different correlation lengths ( $\epsilon=\ell_{c} / L=10, \epsilon=1$, and $\epsilon=0.1$ ) as a function of the numbers of Monte Carlo trials $N$. As in the previous case, the Arlequin estimate depends on both the number of Monte Carlo trials, but also on those realizations themselves, so each value of $\underline{K}_{N}^{\epsilon}$ is computed for $n=10$ different ensembles of realizations of the random medium. In the same Figure 5, we compare the Arlequin estimates with the KUBC and SUBC estimates already presented in Figure 4 and discussed in Section 5.1.2.

The results are extremely convincing in the case presented here. Even when the correlation length is much smaller than the computational cell $(\epsilon=10)$, the iterative Arlequin method predicts the correct homogenized coefficient, in the limit of large number of Monte Carlo realizations. The bias that is observed in the KUBC and SUBC estimates for large $\epsilon$ cancels completely for our estimate. 


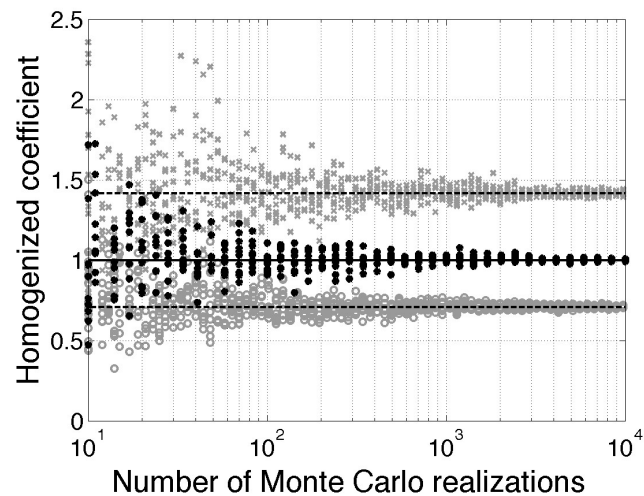

(a) $\epsilon=10$

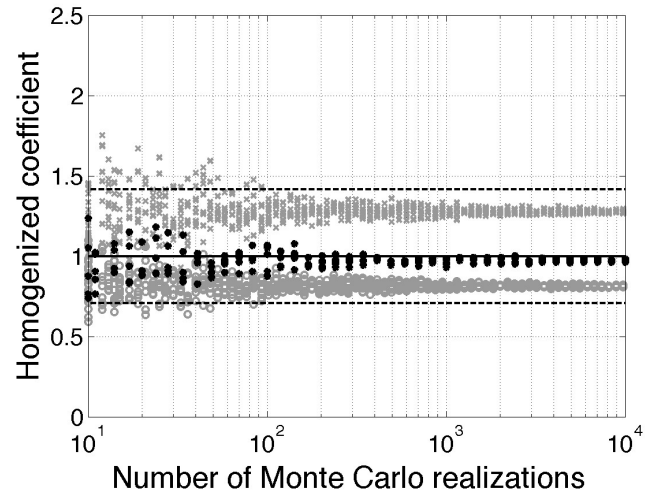

(b) $\epsilon=1$

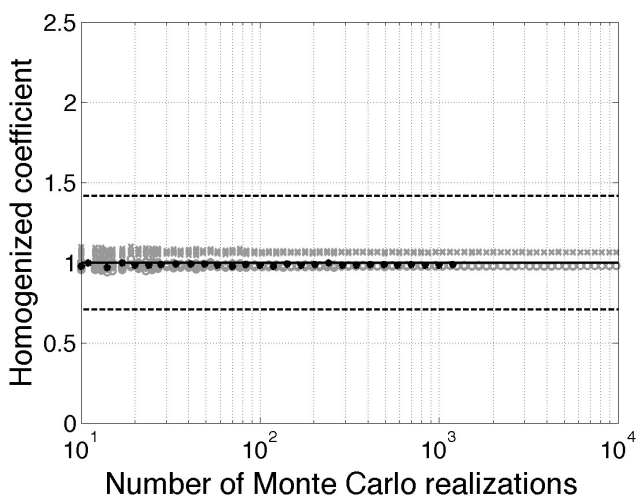

(c) $\epsilon=0.1$

Figure 5. Convergence of the Arlequin estimate $\underline{K}_{N}^{\epsilon}$ (black pluses) for different correlation lengths ((a) $\epsilon=\ell_{c} / L=10$, (b) $\epsilon=1$, and (c) $\epsilon=0.1$ ) as a function of the numbers of Monte Carlo trials $N$, and comparison with the coefficients $\check{K}_{N}^{\epsilon}$ (light grey crosses) and $\hat{K}_{N}^{\epsilon}$ (light grey circles). The dashed lines indicate the values of the arithmetic average $\mathrm{E}\left[\boldsymbol{k}_{\epsilon}\right]$ and of the harmonic average $\mathrm{E}\left[\boldsymbol{k}_{\epsilon}^{-1}\right]^{-1}$. The solid lines indicate the value of $K^{*}=1$.

However, it should be reminded that we have considered here the homogenization of a particular random medium. The random field $\boldsymbol{k}_{\epsilon}$ is indeed locally invariant by inversion, and the homogenized tensor does not depend on the correlation structure. At this point, it therefore cannot be stated unambiguously whether the excellent behavior of our method is a coincidence or a general behavior. In any case, it should be stressed that the KUBC and SUBC approaches behave much worse than our Arlequin estimate.

In the next section, we present a $1 \mathrm{D}$ example, for which we still know analytically the homogenized tensor, but for which the random medium is not locally invariant by inversion. As will be seen, and although the behavior is still much better than for the KUBC, the Arlequin estimate appears slightly biased for that example.

5.1.4. Numerical considerations. Before going over to this $1 \mathrm{D}$ example, we now consider in more detail four numerical issues: (1) the behavior of the potential function over which the minimization problem is solved for the identification of the Arlequin estimate $\underline{K}_{N}^{\epsilon}$ (see algorithm 1), (2) the dependence of the method on the choice of the initial value for the Arlequin estimate, (3) the corresponding number of iterations for convergence, and (4) the cost of the Arlequin method. 
We first compute and plot the value of the potential $\int_{\underline{D}}\left|1-\partial_{x} \underline{u}_{\epsilon}\right| d x$ (which is minimized through the iterative loop of algorithm 1), as a function of the tentative homogenized coefficient $\underline{K}_{\epsilon}$ for the effective model, for a correlation length $\epsilon=\ell_{c} / L=10$, and $n=10$ different ensembles of $N=10^{3}$ realizations of the random medium. As can be observed in Figure 6, this potential seems well adapted for the purpose of pinpointing the right value of the homogenized tensor. It is well behaved, with no local minima and one global minimum. Also, the $n=10$ ensembles of realizations yield similar curves.

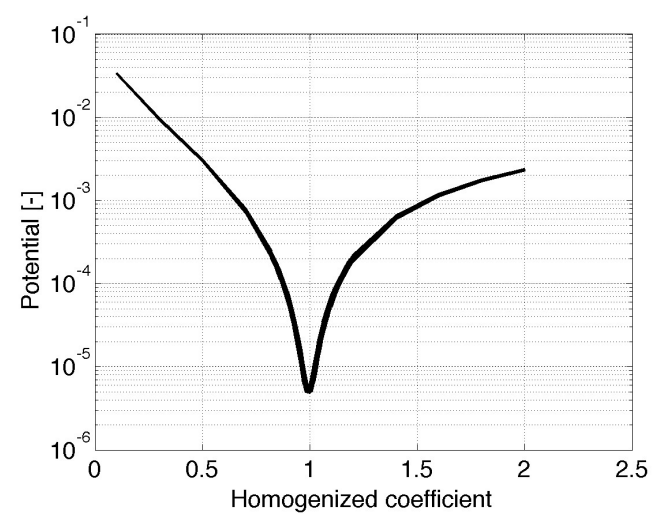

Figure 6. Value of the potential $\int_{\underline{D}}\left|1-\partial_{x} \underline{u}_{\epsilon}\right| d x$ as a function of the coefficient of the tentative homogenized medium, for $\epsilon=10$, and for $n=10$ different ensembles of $10^{4}$ realizations of the random medium.

We then consider the dependence of the method on the choice of the homogenized tensor used to initialize the iterative process of algorithm 1 . We therefore compute estimates of the homogenized tensor for $n=3$ different ensembles of $N=10^{3}$ realizations of a random medium with $\epsilon=1$, for different values of the initial value $K_{0}$. These estimates are grouped in table I, along with the numbers of iterations required for convergence. It is observed that the estimate is independent (to the order of criterion) of the initial value. The only difference lies in the number of iterations required to attain that convergence. Unless one chooses values that are one order of magnitude away from the exact value, the convergence is obtained (for criterion $=10^{-2}$ ) in around 7 iterations.

Table I. Arlequin estimate $\underline{K}_{N}^{\epsilon}$ obtained for different values of the initial coefficient $K_{0}$ initializing the optimization in algorithm 1, and corresponding number of iterations for convergence. The computations are performed for three different ensembles of $N=10^{3}$ Monte Carlo realizations, a correlation length $\epsilon=\ell_{c} / L=1$, and a relative tolerance on both the value and the argument of the potential function of criterion $=10^{-2}$.

\begin{tabular}{|l|c|c|c|c|c|c|}
\hline Initial values & 0.1 & 0.8 & 1.0 & 1.2 & 5.0 & 10.0 \\
\hline \hline Final value & 0.985 & 0.985 & 0.982 & 0.981 & 0.985 & 0.985 \\
\hline Nb iterations & 11 & 6 & 6 & 8 & 11 & 11 \\
\hline \hline Final value & 0.994 & 0.994 & 0.991 & 0.990 & 0.994 & 0.994 \\
\hline Nb iterations & 11 & 6 & 6 & 8 & 11 & 11 \\
\hline \hline Final value & 1.022 & 1.020 & 1.028 & 1.024 & 1.022 & 1.022 \\
\hline Nb iterations & 11 & 6 & 6 & 8 & 11 & 11 \\
\hline
\end{tabular}

We now consider the issue of cost, following on with the discussion at the end of Section 4. Each Arlequin estimate will require the solution of a coupled problem with more degrees of freedom that those involved in the computation of the KUBC and SUBC estimates. Note, however, that we will consider a uniform mesh with intervals of size 0.1 for the entire domain $\underline{D}$, whatever the value of $\epsilon$, and the same meshes as for the KUBC and SUBC computations for the random domain $D$. 
The space dimension of the functional space $\mathcal{W}_{c}$ will be also discretized with elements of size 0.1 . Hence, for $\epsilon=10$, the number of additional degrees of freedom is important compared to the number of degrees of freedom related to $D$, but quickly drops to relatively small numbers for small $\epsilon$. For $\epsilon=0.1$, the number of additional degrees of freedom (in space) is around 1800 for the discretization of $\underline{D}$ and around 200 for the discretization of $\mathcal{W}_{c}$, to be compared to the 10000 degrees of freedom (in space) defined over the random domain $D$. Smaller $\epsilon$ would yield even smaller relative numbers.

\section{2. $1 D$ bar with random properties}

This second example is very similar to the previous one, except that it is one-dimensional.

5.2.1. Description of the model. We now consider a $1 \mathrm{D}$ bar, defined over domain $D=[0 ; 1]$, of length $L=1$, and the homogenization of the operator $-\partial_{x} \boldsymbol{k}_{\epsilon}(x) \partial_{x} \boldsymbol{u}_{\epsilon}$, where $\boldsymbol{k}_{\epsilon}(x)$ is a one-dimensional random heterogeneous modulus. More particularly, we consider a statistically homogeneous random field with uniform first-order marginal density, average $\mathrm{E}\left[\boldsymbol{k}_{\epsilon}\right]=1$, standard deviation $\sigma=1 / 2$ (hence the realizations of $\boldsymbol{k}_{\epsilon}$ are in the interval $[1-\sqrt{3} / 2,1+\sqrt{3} / 2]$ ), triangular spectrum, and correlation length $\ell_{c}$. Note that this random medium is not invariant by inversion. We will consider the homogenization problem for three different relative correlation lengths: $\epsilon=\ell_{c} / L$, $\epsilon=1$ and $\epsilon=0.01$ (see Figure 7 for examples of realizations of the random media considered).

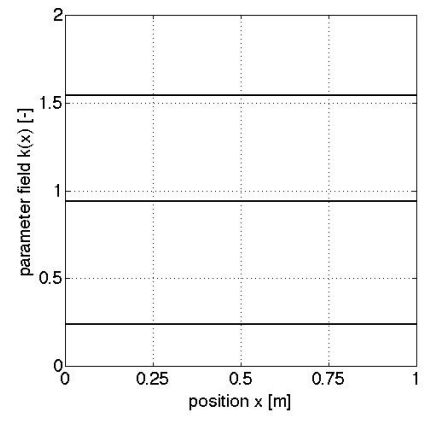

(a) $\epsilon=10$

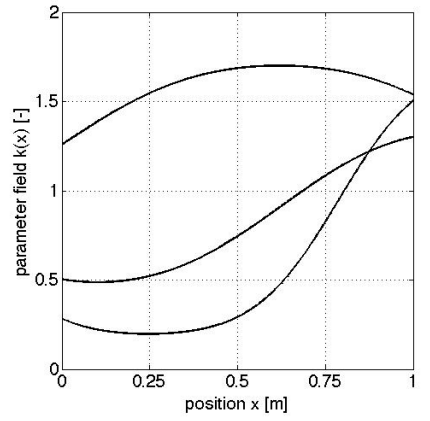

(b) $\epsilon=1$

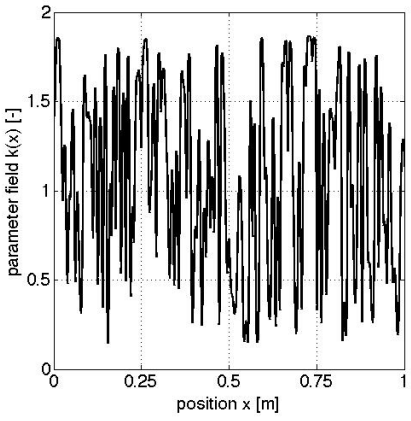

(c) $\epsilon=0.01$

Figure 7. Three realizations of the random field $\boldsymbol{k}_{\epsilon}(x)$ for three correlation lengths ((a) $\epsilon=\ell_{c} / L=0.01$, (b) $\epsilon=1$, and (c) $\epsilon=10)$. For $\epsilon=0.01$, only one realization is plotted for clarity.

For that simple 1D operator, the exact value of the homogenized coefficient can be computed analytically (see Section 2.4):

$$
K^{*}=\mathrm{E}\left[\boldsymbol{k}_{\epsilon}^{-1}\right]^{-1}=\frac{\sqrt{3}}{\ln \left(1+\frac{\sqrt{3}}{2}\right)-\ln \left(1-\frac{\sqrt{3}}{2}\right)} \approx 0.6576 .
$$

5.2.2. Computation of KUBC and SUBC estimates. As in the 2D case (Section 5.1.2), we first consider the KUBC and SUBC estimates of the homogenized coefficient. We compute these estimates for different values of this number $N$ of realizations of the random medium over which averages are taken (equations (7) and (8)), and for different ensembles of $\mathrm{N}$ realizations. The results are plotted in Figure 9. The linear Finite Element method was used to compute the corrector problems, with 4,10, and 1000 elements, respectively for the cases $\epsilon=\ell_{c} / L=10, \epsilon=1$ and $\epsilon=0.01$. On these plots, we retrieve the expected asymptotic behavior of the homogenized coefficients.

5.2.3. Computation of the Arlequin estimate. We now turn to the estimation of the Arlequin estimate $\underline{K}_{N}^{\epsilon}$ of the homogenized coefficient over domains $\underline{D}=[-1,2]$ and $D=[0,1]$. The coupling area, over which both models exchange information, is $D_{c}=[0,0.2] \cup[0.8,1]$. The 
boundary conditions that we consider are $\underline{u}_{\epsilon}(x=-1)=-1$ and $\underline{u}_{\epsilon}(x=2)=2$, which correspond to a unit strain boundary condition (Dirichlet approach). As in the $2 \mathrm{D}$ case, we continue to define $\epsilon$ as the ratio of the correlation length $\ell_{c}$ to the size $L$ of the random cell $D$ (rather than to the size $\underline{L}=3$ of $\underline{D}$ ), because it is $L$ that actually indicates the amount of statistical information available on the random medium.

In Figure 8, we plot the values of the Arlequin estimate $\underline{K}_{N}^{\epsilon}$ as a function of the numbers of Monte Carlo trials $N$. Each value of $\underline{K}_{N}^{\epsilon}$ is computed for $n=10$ different ensembles of realizations of the random medium. In the same Figure 8, we compare the Arlequin estimates with the KUBC and SUBC estimates and with the theoretical value of the homogenized coefficient $K^{*} \approx 0.6576$.

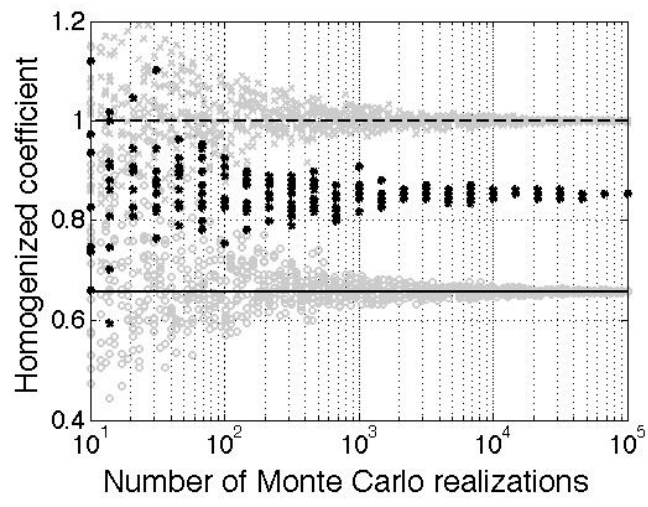

(a) $\epsilon=10$

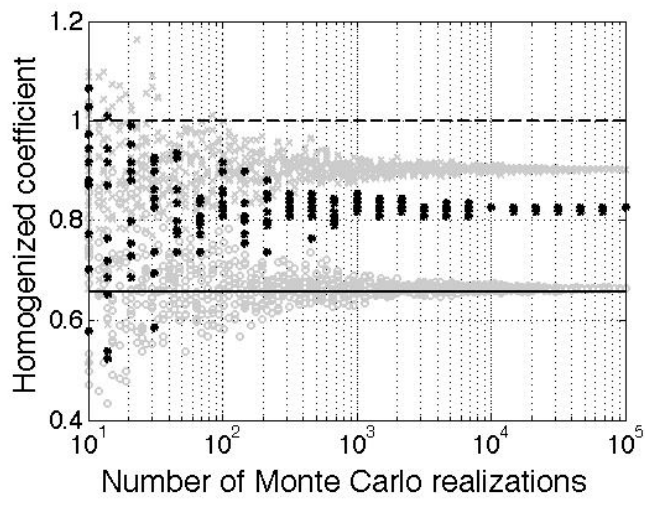

(b) $\epsilon=1$

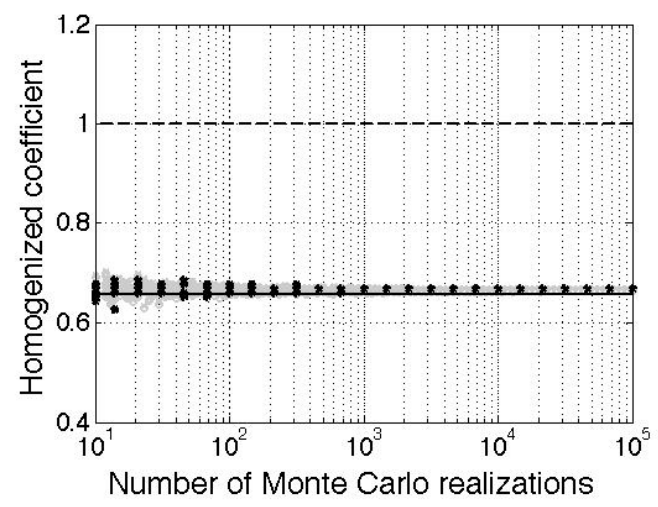

(c) $\epsilon=0.01$

Figure 8. Convergence of the Arlequin estimate $\underline{K}_{N}^{\epsilon}$ (black pluses) for different correlation lengths ((a) $\epsilon=\ell_{c} / L=10$, (b) $\epsilon=1$, and (c) $\epsilon=0.01$ ) as a function of the numbers of Monte Carlo trials $N$, and comparison with the coefficients $\check{K}_{N}^{\epsilon}$ (light grey crosses) and $\hat{K}_{N}^{\epsilon}$ (light grey circles). The dashed lines indicate the values of the arithmetic average $\mathrm{E}\left[\boldsymbol{k}_{\epsilon}\right]$ and the solid lines indicate the value of the harmonic average $\mathrm{E}\left[\boldsymbol{k}_{\epsilon}^{-1}\right]^{-1}=K^{*}$.

As can be observed, and as already announced at the end of Section 5.1, our approach provides better estimates than the classical KUBC (the SUBC is exact in 1D). However, the results are not perfectly unbiased. Note also that, if we had used Neumann boundary conditions for our Arlequin estimate (results not shown), we would have obtained the same results as the SUBC.

To refine these observations, we present in Figure 9 the Arlequin estimates obtained for $N=10^{3}$ Monte Carlo trials as a function of the correlation length $\epsilon=\ell_{c} / L$. Again, each experiment is repeated for $n=10$ different ensembles of realizations of the random medium. It seems that the bias of the Arlequin estimate drops down to close to zero for $\epsilon$ of the order of 0.1. Finally, several numerical tests were considered, as in Section 5.1.4, and similar results were obtained (not shown): 


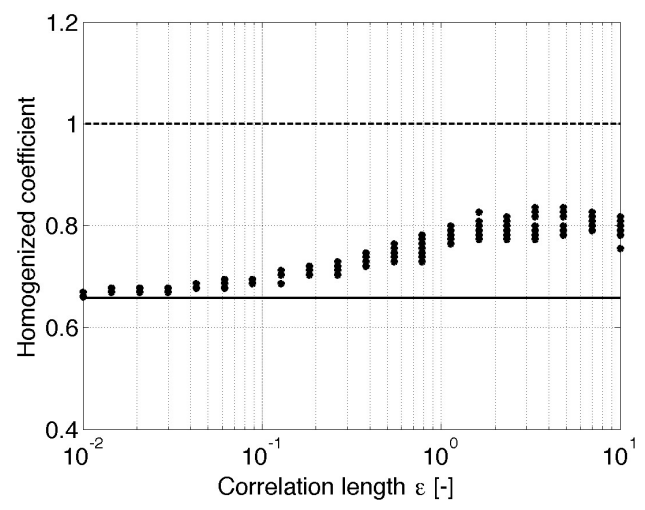

Figure 9. Convergence of the Arlequin estimate $\underline{K}_{N}^{\epsilon}$ as a function of the correlation length $\epsilon=\ell_{c} / L$, for different ensembles of $N=10^{3}$ realizations of the random medium. The dashed line indicates the value of the arithmetic average $\mathrm{E}\left[\boldsymbol{k}_{\epsilon}\right]$ and the solid line indicates the value of the harmonic average $\mathrm{E}\left[\boldsymbol{k}_{\epsilon}^{-1}\right]^{-1}=K^{*}$.

the shape of the potential function is adequate and the number of iterations ranges from 6 to 11 , depending on the initial value chosen for the homogenized coefficient (from 0.1 to 10).

\section{3. $2 D$ periodized bi-phasic material with spherical inclusions}

This last example aims, on the first hand, to present an example with discontinuous properties, and, on the other hand, to compare the behavior of the method proposed here to the classical method of periodic homogenization.

5.3.1. Description of the model. We consider, as in the first example, a domain $D=[0,1] \times[0,1]$, of typical size $L=1$. This domain is separated into a matrix and overlapping (almost) spherical inclusions, with diameter $\ell_{c}$. The operator to be homogenized is $\nabla \cdot \boldsymbol{k}_{\epsilon}(x) \nabla \boldsymbol{u}_{\epsilon}$, where $\boldsymbol{k}_{\epsilon}(x)$ is equal to 1 in the matrix and 10 in the inclusions. The centers of the spheres are uniformly distributed in a larger domain $([-10,10] \times[-10,10])$, with an average concentration of $c=0.3$. The computational domain is then periodized, that is to say the centers inside the computational cell $D$ are repeated outside of it before the spheres are constructed. We will consider the homogenization problem for four different relative correlation lengths: $\epsilon=\ell_{c} / L=8 / 3>\sqrt{2}$ (for which periodization implies that all the realizations are homogeneous, $30 \%$ of them with value 10 and $70 \%$ of them with value 1 ), $\epsilon=2 / 3, \epsilon=1 / 3$, and $\epsilon=1 / 6$ (which correspond, on average, to 1,4 and 16 spheres in each computational cell, respectively). Examples of realizations of the random media considered can be observed on Figure 10. Note that the discretization of the spheres is exaggerated in the smaller cells in order not to keep the shape of the inclusions exactly the same (up to homothety) in all the cases considered. This is to avoid the introduction of a bias due to a modification of the shape of the inclusions with size.

5.3.2. Computation of KUBC, SUBC and periodic estimates. The KUBC, SUBC and periodic estimates are computed for different values of the number $N$ of realizations of the random medium over which averages are taken and, each time, for $n=5$ different ensembles of $\mathrm{N}$ realizations. The results are plotted in Figure 11. The linear Finite Element method was used to compute the corrector problems, with $288,576,1152$, and 2304 triangular elements, respectively for the cases $\epsilon=\ell_{c} / L=8 / 3, \epsilon=2 / 3, \epsilon=1 / 3$, and $\epsilon=1 / 6$.

5.3.3. Computation of the Arlequin estimate and discussion. The Arlequin estimate $\underline{K}_{N}^{\epsilon}$ of the homogenized coefficient is computed over domains $\underline{D}=[-1,2] \times[-1,2]$ and $D=[0,1] \times[0,1]$. The coupling zone $D_{c}$ is a band of width 0.4 circling at the boundary of $D$, and we consider a unit strain boundary condition at the boundary of $\underline{D}$. For each number $N$ of Monte Carlo realizations, 


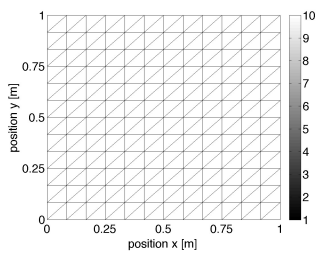

(a) $\epsilon=8 / 3$, Sample 1

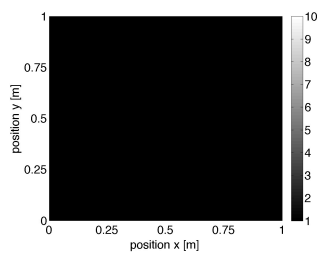

(e) $\epsilon=8 / 3$, Sample 2

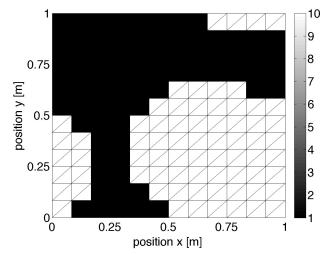

(b) $\epsilon=2 / 3$, Sample 1

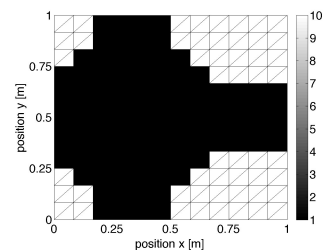

(f) $\epsilon=2 / 3$, Sample 2

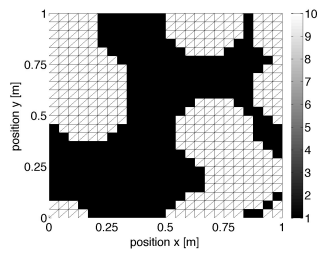

(c) $\epsilon=1 / 3$, Sample 1

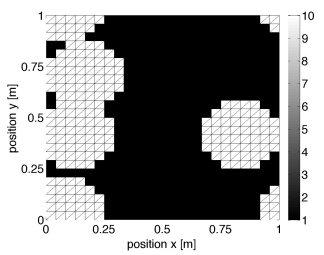

(g) $\epsilon=1 / 3$, Sample 2

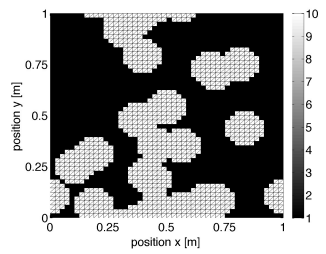

(d) $\epsilon=1 / 6$, Sample 1

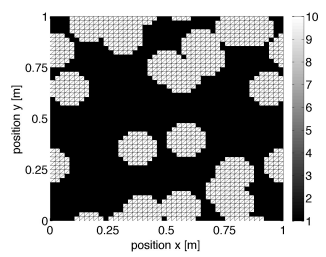

(h) $\epsilon=1 / 6$, Sample 2

Figure 10. Map of parameter $\boldsymbol{k}_{\epsilon}(x)$ for two realizations of each of the cases considered here (from left to right: $\epsilon=\ell_{c} / L=8 / 3, \epsilon=2 / 3, \epsilon=1 / 3$, and $\left.\epsilon=1 / 6\right)$.

Arlequin estimates are computed for 5 different ensembles of $N$ realizations. The number of triangular finite elements for the micro-structure is the same as for the KUBC, SUBC and periodic estimates (see above), and the macro-structure is discretized with 81 elements. In Figure 11, the value of the Arlequin estimate can be observed and compared to those obtained with the KUBC, SUBC, and periodic estimates.

The first observation that should made on these results is that, except for the smallest cell (Figure 11(a)), the estimates obtained with our method and with the periodic boundary conditions are very similar. The KUBC and SUBC estimates provide bounds for both these estimates (at convergence for a given cell size). The second observation concerns the first case. Remember that periodization of the medium implies that the realizations for $\epsilon=8 / 3$ are all homogeneous. In that case, all knowledge of correlation is lost, and only the first-order marginal law remains. As the realizations are all homogeneous, the periodic boundary conditions therefore provide exactly the same estimates as the KUBC, which are very bad. On the other hand, the Arlequin estimate provides a reasonable value of the homogenized coefficient. Note however that, as all statistical information has disappeared, except that related to the first-order marginal law, we do not expect that the Arlequin method will in general provide perfect estimates of the homogenized coefficient. As before, this example should be seen as a promising feature of our method.

\section{CONCLUSIONS AND PROSPECTS}

In this paper, we have introduced a new computational method for the homogenization of random media. It is based on two major ingredients: (1) a stochastic-deterministic coupling method that limits the influence of the boundary conditions in the homogenization experiments, and (2) an iterative technique for updating the value of the tentative deterministic model. At convergence, the material parameter of the deterministic model is expected to provide the value of the homogenized tensor. The results obtained for the chosen $2 \mathrm{D}$ example are spectacular. In that case, the bias observed in the KUBC and SUBC estimates totally disappears, even for very large correlation length $\epsilon=\ell_{c} / L$. On the other hand, the biases obtained in the 1D example are non-zero but smaller than for the corresponding KUBC estimates. With respect to the classical periodic homogenization method, our estimates seem to compare well at all sizes, except for small cells, for which our estimates are much more accurate.

This promising behavior of the proposed method should obviously be confirmed on other examples, starting with a $3 \mathrm{D}$ problem. However, this method will show its true interest not on 


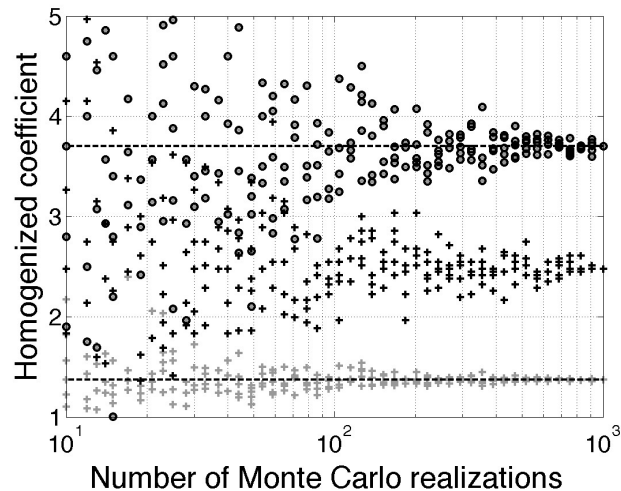

(a) $\epsilon=8 / 3$

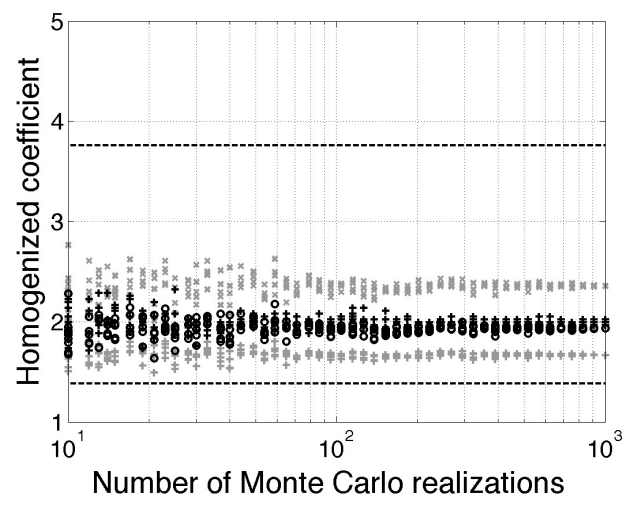

(c) $\epsilon=1 / 3$

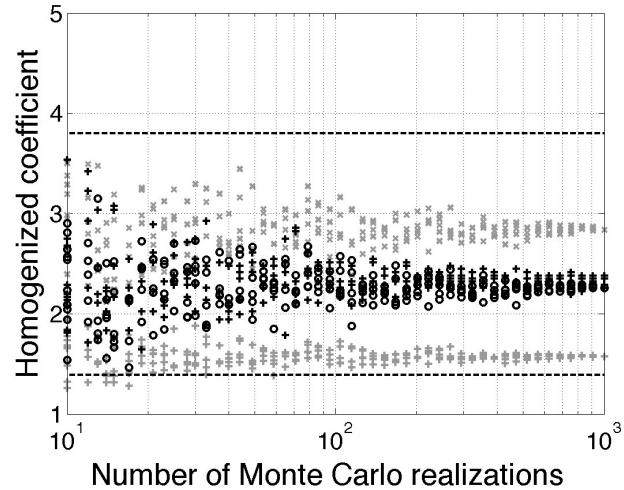

(b) $\epsilon=2 / 3$

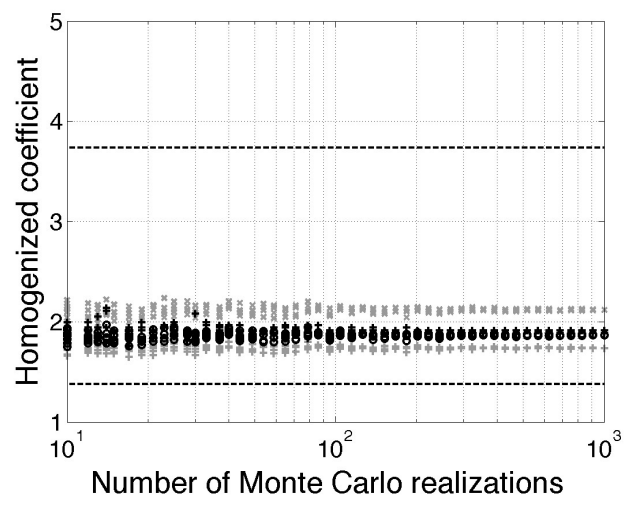

(d) $\epsilon=1 / 6$

Figure 11. Convergence of the Arlequin estimate $\underline{K}_{N}^{\epsilon}$ (black pluses) for different correlation lengths ((a): $\epsilon=\ell_{c} / L=8 / 3$, (b): $\epsilon=2 / 3$, (c): $\epsilon=1 / 3$, and (d): $\left.\epsilon=1 / 6\right)$ as a function of the numbers of Monte Carlo trials $N$, and comparison with the coefficients $\check{K}_{N}^{\epsilon}$ (light grey crosses) and $\hat{K}_{N}^{\epsilon}$ (light grey pluses) and the periodc estimate (black circles). The dashed lines indicate the values of $\mathrm{E}\left[\boldsymbol{k}_{\epsilon}\right]$ and $\mathrm{E}\left[\boldsymbol{k}_{\epsilon}^{-1}\right]^{-1}$.

examples (as those that were considered in this paper) for which current techniques already work (in particular the periodic boundary conditions approach), but really on examples where the full potential of the Arlequin method as a multi-model coupling technique can be used. In particular, the approach can be used in cases when the equation driving the behavior of the micro-structure is not the same as the equation driving the homogenized equation. As an example, one could consider the homogenization of an elastic random media by a beam model. Other promising examples include the coupling of wave propagation models with kinetic models (where the variable of interest is not a displacement field but a phase-space energy density) [38, 39].

\section{ACKNOWLEDGEMENTS}

This work was partially supported by the ANR project TYCHE (Advanced methods using stochastic modeling in high dimension for uncertainty modeling, quantification and propagation in computational mechanics of solids and fluids), with project number ANR-2010-BLAN-0904, and by grants from DIGITEO and Région Ile-de-France, with project number 2009-26D. The author would also like to thank Prof. Didier Clouteau for fruitful discussions on the content of this paper.

\section{REFERENCES}


1. Papanicolaou GC, Varadhan SR. Boundary value problems with rapidly oscillating random coefficients. Proceedings of the Conference on Random Fields, Seria Colloquia Mathematica Societatis Janos Bolyai, vol. 2, Fritz J, Lebowitz JL (eds.), North Holland, 1981; 835-873.

2. Tartar L. The general theory of homogenization: a personalized introduction, Lecture notes of the Unione Matematica Italiana, vol. 7. Springer, 2009.

3. Kozlov SM. Averaging of random operators. Math. USSR Sbornik 1980; 37(2):167-180, doi:10.1070/ SM1980v037n02ABEH001948.

4. Zhikov VV, Kozlov SM, Oleinik OA, Ngoan KT. Averaging and G-convergence of differential operators. Russian Math. Surv. 1979; 34:69, doi:10.1070/RM1979v034n05ABEH003898.

5. Dal Maso G, Modica L. Nonlinear stochastic homogenization. Annali di Matematica Pura ed Applicata 1986; 144(1):347-389, doi:10.1007/BF01760826.

6. Yurinskii VV. Averaging elliptic equations with random coefficients. Siberian Math. J. 1979; 20(4):611-623, doi: 10.1007/BF00970374.

7. Yurinskii VV. Averaging an elliptic boundary-value problem with random coefficients. Siberian Math. J. 1980; 21(3):470-482, doi:10.1007/BF00968192.

8. Sab K. On the homogenization and the simulation of random materials. Europ. J. Mech. A/Solids 1992; 11(5):585607.

9. Bourgeat A, Mikelić A, Wright S. Stochastic two-scale convergence in the mean and applications. J. Reine Angewandte Math. 1994; 1994(456):19-52, doi:10.1515/crll.1994.456.19.

10. Bourgeat A, Kozlov SM, Mikelić A. Effective equations of two-phase flow in random media. Calc. Var. Part. Diff. Equ. 1995; 3(3):385-406, doi:10.1007/BF01189397.

11. Lejay A. Homogenization of divergence-form operators with lower-order terms in random media. Prob. Theory Related Fields 2001; 120(2):255-276, doi:10.1007/PL00008783.

12. Caffarelli LA, Souganidis PE, Wang L. Homogenization of fully nonlinear, uniformly elliptic and parabolic partial differential equations in stationary ergodic media. Comm. Pure Appl. Math. 2005; 58(3):319-361, doi: 10.1002/cpa.20069.

13. Blanc X, Le Bris C, Lions PL. Stochastic homogenization and random lattices. Journal de Mathématiques Pures et Appliquées 2007; 88(1):34-63, doi:10.1016/j.matpur.2007.04.006.

14. Le Bris C. Some numerical approaches for weakly random homogenization. Numerical Mathematics and Advanced Applications 2009, et al GK (ed.), Springer-Verlag, 2010; 29-45, doi:10.1007/978-3-642-11795-43.

15. Bourgeat A, Piatnitski A. Approximations of effective coefficients in stochastic homogenization. Ann. I. H. Poincaré 2004; 40:153-165, doi:10.1016/j.anihpb.2003.07.003.

16. Kanit T, Forest S, Galliet I, Mounoury V, Jeulin D. Determination of the size of the representative volume element for random composites: statistical and numerical approach. Int. J. Solids. Struct. 2003; 40:3647-3679.

17. Povirk GL. Incorporation of microstructural information into models of two-phase materials. Acta Metall. Mater. 1995; 43(8):3199-3206, doi:10.1016/0956-7151(94)00487-3.

18. Gusev AA. Representative volume element size for elastic composites: a numerical study. J. Mech. Phys. Solids 1997; 45(9):1449-1459, doi:10.1016/S0022-5096(97)00016-1.

19. Roberts AP, Garboczi EJ. Elastic properties of model porous ceramics. J. Amer. Ceramic Soc. 2000; 83(12):30413048, doi:10.1111/j.1151-2916.2000.tb01680.x.

20. Zeman J, Šejnoha M. Numerical evaluation of effective elastic properties of graphite fiber tow impregnated by polymer matrix. J. Mech. Phys. Solids 2001; 49(1):69-90, doi:10.1016/S0022-5096(00)00027-2.

21. Meille S, Garboczi EJ. Linear elastic properties of 2D and 3D models of porous materials made from elongated objects. Mod. Simul. Mat. Sci. Engrg. 2001; 9(5):371, doi:10.1088/0965-0393/9/5/303.

22. Costaouec R, Le Bris C, Legoll F. Variance reduction in stochastic homogenization: proof of concept, using antithetic variables. Boletin Sociedad Espa nola Matematica Aplicada 2010; 50:9-27.

23. Héraud S, Allais L, Haddadi H, Marini B, Teodosiu C, Zaoui A. Du polycristal au multicristal: vers un mésoscope numérique. J. de Physique IV 1998; 8(Pr4):27-32, doi:10.1051/jp4:1998403.

24. Haddadi H, Teodosiu C, Héraud S, Allais L, Zaoui A. A "numerical mesoscope" for the investigation of local fields in rate-dependent elastoplastic materials at finite strain. IUTAM Symposium on Computational Mechanics of Solid Materials at Large Strains, Miehe C (ed.), Kluwer Academic Publishers, 2003; 311-320.

25. Cottereau R, Ben Dhia H, Clouteau D. Localized modeling of uncertainty in the Arlequin framework. IUTAM Symposium on the Vibration Analysis of Structures with uncertainties, Belyaev A, Langley R (eds.), IUTAM Bookseries, Springer, 2010; 477-488, doi:10.1007/978-94-007-0289-9_33.

26. Cottereau R, Clouteau D, Ben Dhia H, Zaccardi C. A stochastic-deterministic coupling method for continuum mechanics. Comp. Meth. Appl. Mech. Engrg. 2011; 200(47-48):3280-3288, doi:10.1016/j.cma.2011.07.010.

27. Huet C. Application of variational concepts to size effects in elastic heterogeneous bodies. J. Mech. Phys. Solids 1990; 38(6):813-841, doi:10.1016/0022-5096(90)90041-2.

28. Sab K, Nedjar B. Periodization of random media and representative volume element size for linear composites. Comptes-Rendus Mecanique 2005; 333:187-195, doi:10.1016/j.crme.2004.10.003.

29. Moulinec H, Suquet P. A numerical method for computing the overall response of nonlinear composites with complex microstructures. Comp. Meth. Appl. Mech. Engrg. 1998; 157:69-94.

30. Milton GW. The theory of composites. Cambridge Monographs on Applied and Computational Mechanics, Cambridge University Press, 2002.

31. Ben Dhia H. Multiscale mechanical problems: the Arlequin method. Comptes Rendus de l'Académie des Sciences - Series IIB 1998; 326(12):899-904.

32. Ben Dhia H, Rateau G. Mathematical analysis of the mixed Arlequin method. Comptes Rendus Acad. Sci. - Series I - Math. 2001; 332(7):649-654, doi:10.1016/S0764-4442(01)01900-0.

33. Ben Dhia H, Rateau G. The Arlequin method as a flexible engineering design tool. Int. J. Numer. Meths. Engr. 2005; 62(11):1442-1462. 
34. Ben Dhia H. Further insights by theoretical investigations of the multiscale Arlequin method. Int. J. Multiscale Comp. Engr. 2008; 6(3):215-232.

35. Prudhomme S, Chamoin L, Ben Dhia H, Bauman PT. An adaptive strategy for the control of modeling error in two-dimensional atomistic-to-continuum coupling simulations. Comp. Meth. Appl. Mech. Engrg. 2009; 198(2126):1887-1901, doi:10.1016/j.cma.2008.12.026.

36. Lagarias J, Reeds JA, Wright MH, Wright PE. Convergence properties of the Nelder-Mead simplex method in low dimensions. SIAM J. Optim. 1998; 9(1):112-147.

37. Shinozuka M, Deodatis G. Simulation of stochastic processes by spectral representation. Appl. Mech. Rev. 1991; 44(4):191-205, doi:10.1115/1.3119501.

38. Ryzhik L, Papanicolaou G, Keller JB. Transport equations for elastic and other waves in random media. Wave Motion 1996; 24:327-370.

39. Ta QA, Clouteau D, Cottereau R. Modeling of random anisotropic elastic media and impact on wave propagation. Europ. J. Comp. Mech. 2010; 19(1-3):241-253, doi:10.3166/ejcm.19.241-253. 